\title{
INTRAORAL CYSTICERCOSIS OF THE MASSETER MUSCLE- A RARE FINDING; COR- RELATION OF HISTOPATHOLOGY WITH MRI AND USG
}

\author{
M Shrestha ${ }^{1 *}$, P Shakya $^{2}$, S Shrestha ${ }^{3}$ \\ ${ }^{1} 1$ Department of Oral \& Maxillofacial Pathology, Chitwan Medical College,College of Dental Sciences,Bharatpur-10, Chitwan, Nepal. \\ ${ }^{2}$ Department of Oral \& Maxillofacial Surgery, Peoples Dental College \& Hospital, Nayabazaar, Kathmandu, Nepal. \\ ${ }^{3}$ Department of Oral \& Maxillofacial Surgery,Nepal Police Hospital,Kathmandu, Nepal. \\ *Correspondence to: Dr Madhu Shrestha, Department of Oral \& Maxillofacial Pathology, Chitwan Medical College, \\ College of Dental Sciences, Bharatpur-10, Chitwan, Nepal. \\ Email:drmadhushrestha@gmail.com
}

\begin{abstract}
Cysticercosis refers to infection of tissues after exposure of eggs of pork tapeworm Taenia solium. The authors report a rare case of a solitary intraoral cysticercosis in a thirty-four year female who presented with a mass in the left buccal mucosa of two months duration. The attending dentist could not have an impression of the lesion and sent for ultrasonography. The ultrasonographic report described a well defined hypoechoic lesion with central hypoechoic mural nodular area in left buccal region with muscle plane along with posterior enhancement suggestive of cystic nature. The impression was of benign cystic lesion in left buccal region (muscle plane). The MRI report revealed a $12 \mathrm{~mm}$ diameter T2 high signal intensity area noted in the anterior aspect of the left masseter muscle that showed iso to low signal in T1 weighted images. An ill marginated T2 high signal intensity was seen in left masseter muscle which also showed slight enlargement. The impression was of cystic lesion in left masticator space with edema in surrounding soft tissue plane. The lesion was enucleated and the histological findings revealed a cystic lesion containing parasitic larva. The cystic cavity was surrounded by a collagenous capsule of multinucleated foreign body giant cells with admixture of lymphocytes, plasma cells and histiocytes. The parasitic larva showed thick integument with projections and a myxomatous inner stroma. Thus a definitive diagnosis of "oral cysticercosis" was given. Oral cysticercosis rarely involves the oral cavity. This case reports a rare involvement of cysticercosis involving the masseteric space.
\end{abstract}

Key Words: Masseter, Oral cysticerscosis, Parasitic cyst.

\section{INTRODUCTION}

Cysticercosis refers to infection of tissues after exposure of eggs of the pork tapeworm Taenia solium. This is primarily a foodborne disease that spreads due to ingestion of contaminated food mainly the pork meat. This condition often occurs as a result of poor sanitation and hygiene such as ingestion of contaminated food or polluted drinking water, eating with unclean hands, consumption of raw or inadequately cooked pork1.

After ingestion of the eggs, they pass into the lumen of intestine, from where the larva migrate to other areas such as brain and muscles where they elicit a foreign body reaction that causes cyst formation known as cysticercosis, if this occurs in brain, it is known as neurocysticercosis 2 . They may remain asymptomatic for years as cysts or some cases may cause severe consequences such as seizures in the brain or painful nodules in the muscles 3 . Although many cases of neurocysticersocis have been reported, cysticercosis involving the muscles is considered to be very rare (only nine cases reported to the author's knowledge) 4, 5, 6 .

Cysticercosis only rarely involves the oral cavity and intramuscular involvement in the oral cavity is much rarer. A MEDLINE search revealed only 38 cases of oral cysticercosis published in the English literature 7, 8, 9, 10, 11, 12. Subcutaneous tissue and muscle are the most common sites of involvement. In the oral region, the tongue is most commonly involved, followed by the upper and lower lips, the oral mucosa, and the submandibular and submental tissue.

\section{CASE REPORT}

A thirty four year old female presented with a mass on the left cheek of two months duration. The mass could be felt by the patient with her tongue and had recently developed pain even without touching. No other nodule was evident in any parts of her body.

On examination the lesion was present in the left side buccal vestibule around the third molar region in the maxillary plane of muscles. The mass was oval in shape with a shiny, whitishyellow colour and measured $1.5 \mathrm{X} 1 \mathrm{cms}$. It was bimanually palpable, was tender on palpation, and was movable and soft in consistency. The patient was sent for an ultrasonography by the attending dentist.

The ultrasonographic report described a well defined hypoechoic lesion with central hypoechoic mural nodular area in left buccal region with muscle plane along with posterior enhancement 
suggestive of cystic nature (Figure 1). The impression was of benign cystic lesion in left buccal region (muscle plane) and a differential diagnosis of paracytic cyst was given.

After complete examination of the patient and evaluation in the department of Oral Medicine and Radiology, the patient was further sent for an MRI examination for further confirmation. The MRI report revealed a $12 \mathrm{~mm}$ diameter T2 high signal intensity area noted in the anterior aspect of the left masseter muscle that showed iso to low signal in T1 weighted images. An ill marginated $\mathrm{T} 2$ high signal intensity was seen in left masseter muscle which also showed slight enlargement. The impression was of cystic lesion in left masticator space with edema in surrounding soft tissue plane indicating an inflammatory lesion (Figure 2).

The lesion was a well capsulated at the time of surgery thus was enucleated completely and sent for histopathological examination (Figure 3 and 4). The histological findings revealed a cystic lesion containing degenerated parasitic larva (Figure 5). The cystic cavity was surrounded by a collagenous capsule that comprised of multinucleated foreign body giant cells with admixture of lymphocytes, plasma cells and histiocytes. The parasitic larva showed thick integument with projections and a myxomatous inner stroma (Figure 6). Based on the histopathological findings a definitive diagnosis of "oral cysticercosis" was given. The edema finding in MRI correlated well with the granulomatous inflammation surrounding the parasitic cyst.

The patient was treated with Albendazole after the established diagnosis. Patient was recalled for follow up and no nodule elsewhere has been noticed thereafter.

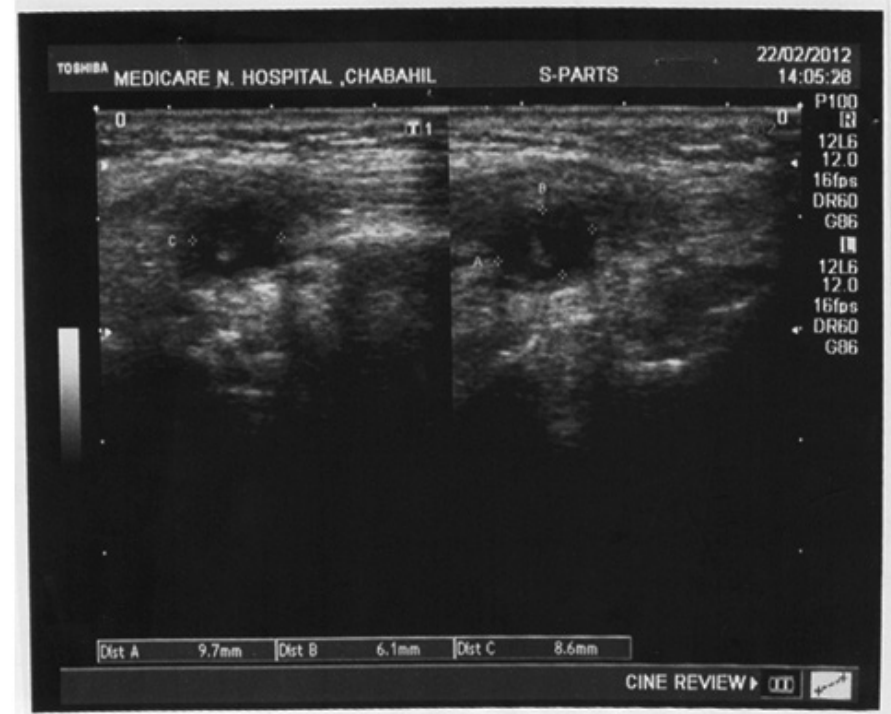

Figure 1: USG of the masseter showing well defined hypoechoic lesion

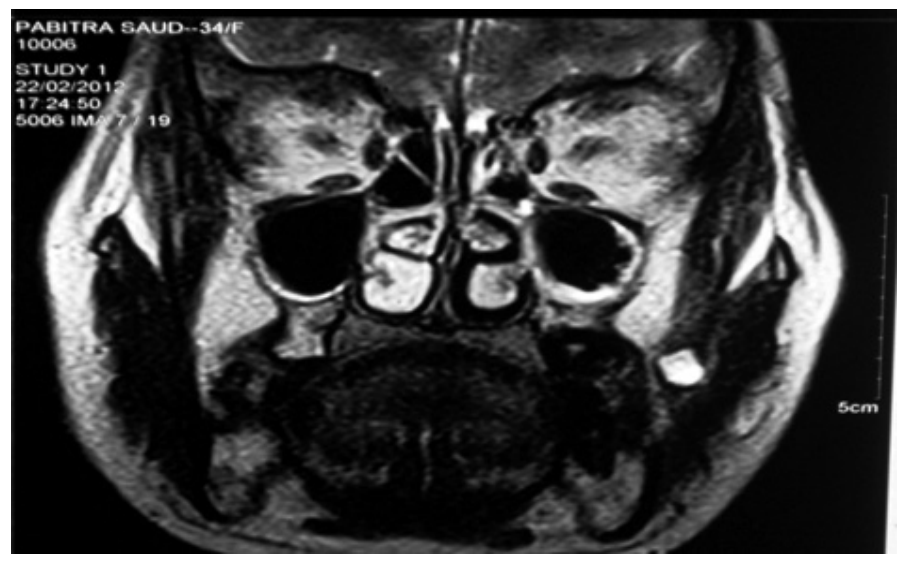

Figure 2: MRI showing $12 \mathrm{~mm}$ diameter T2 high signal intensity area noted in the anterior aspect of the left masseter muscle that showed iso to low signal in $\mathrm{T} 1$ weighted images

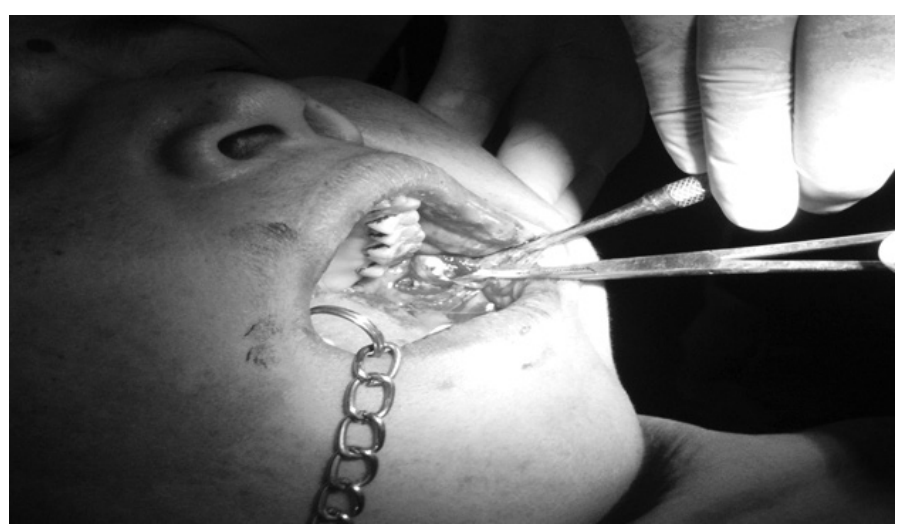

Figure 3: Clinical picture showing the location of the lesion and surgical exploration revealed a shiny capsule.

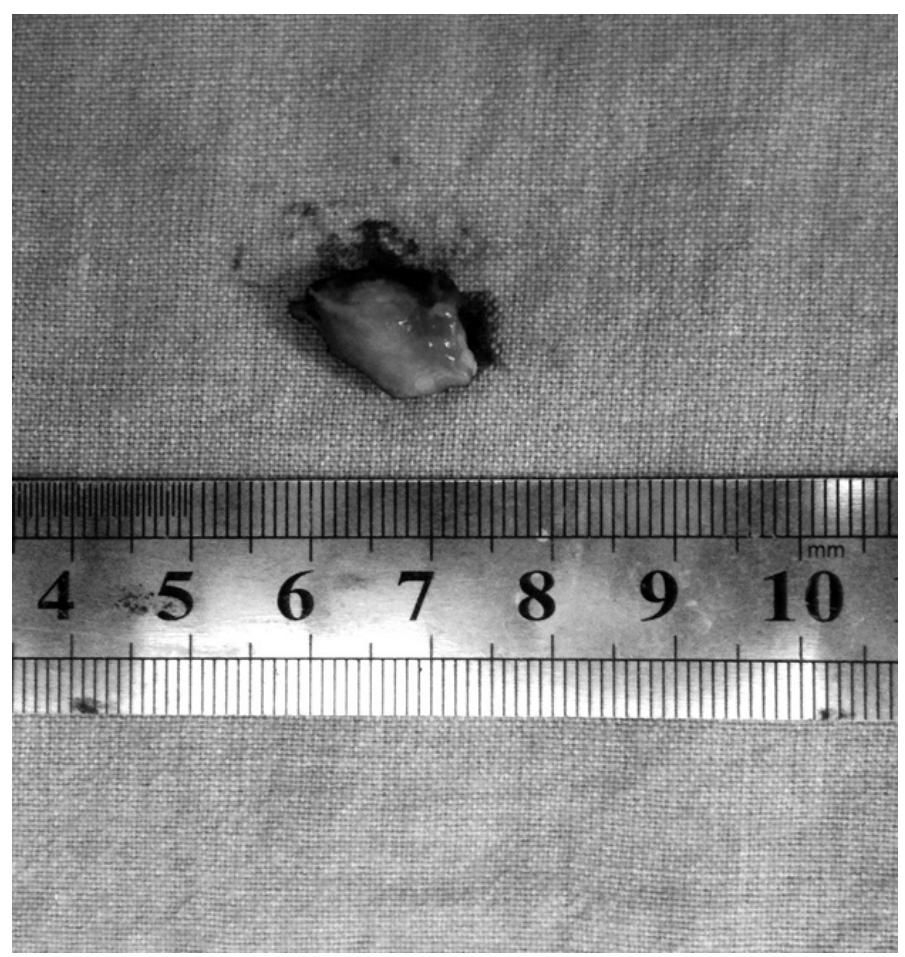

Figure 4: Histopathological specimen 


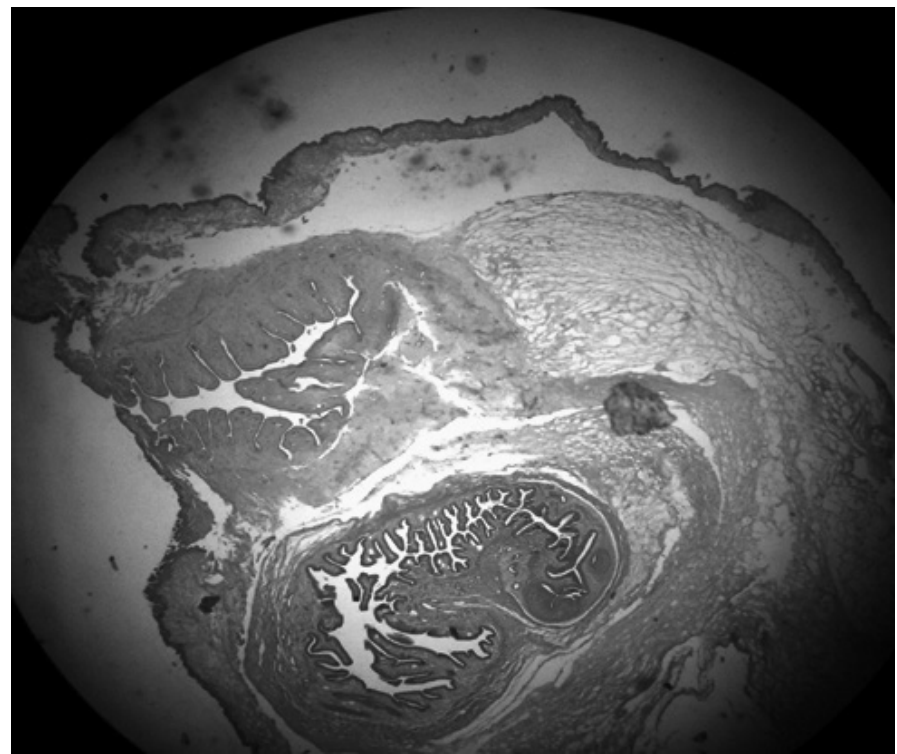

Figure 5: Low power view (4x) showing the parasitic cyst surrounded by granulomatous (arrow) collagenous capsule

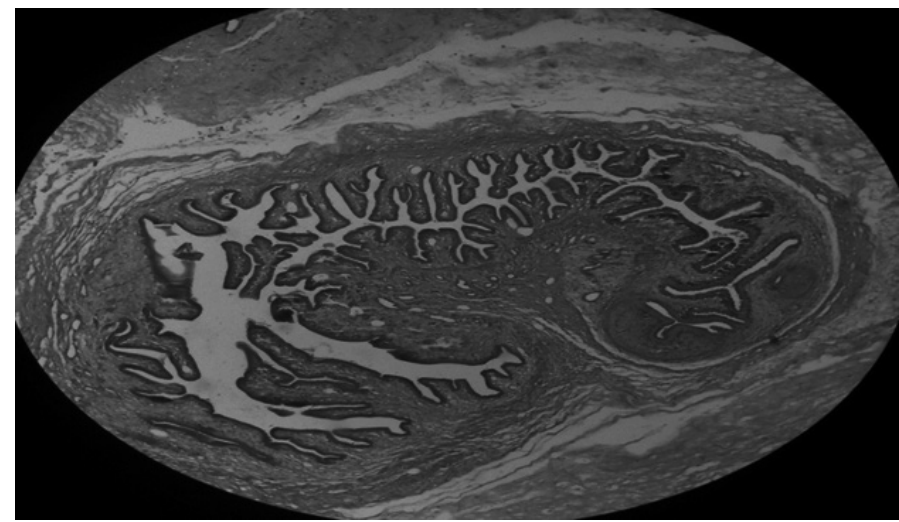

Figure 6: High power view (10x) showing the parasitic larva with thick integuments

\section{DISCUSSION}

Cysticercosis is one of the major public health problems in many developing countries like Russia, China, India, Pakistan, Philippines, Indonesia, and Mexico including Nepal due to endemicity of Pork tapeworm (Taenia solium) in these areas.$^{10,11}$

However, in the context of Nepal, not only the ingestion of larva through pork meat but also poor sanitary condition and use of fecal contaminated water have led to the ingestion of eggs through raw vegetables and fruits also.

The prevalence of oral cysticercosis is not exactly known. Saran et al. reported a prevalence of $4.2 \%$ in 120 cases while Mazhari et al reported 5.2\% in 153 cases. In the context of Nepal, Birendra et al reported sixty-two oral cases out of twenty-three thousand four hundred and two cases ${ }^{1,9,13}$ It can develop in almost any tissue of the body, and clinical manifestation reflects the organ involved. The most common clinical problem is the alteration of central nervous system function as neurocysticercosis. Fineneedle aspiration cytology is a good method for the diagnosis, but a final diagnosis should be made histopathologically. The present case involved diagnosis with histopathological examination along with correlation with the MRI and USG findings. ${ }^{14}$

The incidence of Cysticercosis in the oral cavity has been reported as unusual, as observed in a study by Elias et al, who found only sixty-five cases reported in literature. Saran et $\mathrm{al}^{9}$ analyzing 120 cases, observed $4.2 \%$ in the mouth. Both found a greater incidence in the tongue, followed by labial mucosa and buccal mucosa, and the submandibular and submental tissue. ${ }^{9,10,12,15}$

In an extensive histopathological study of 62 biopsies of cysticercosis in Nepal out of 23,402 biopsy cases, the oral mucosal involvement was of 6 cases $(10 \%)$ only which show the rarity of involvement of the oral cavity. The present case involved a cysticercosis of the oral cavity involving the masseter muscles which is a rare finding. The present case was seen in thirty year female and it correlated with the young age group female population seen in an extensive review study of cysticercosis in Nepal. ${ }^{13}$ Most of the cases reported had asymptomatic history, while in this case the patient had a history of pain and tenderness in the region of muscles that correlates with a report by Sirikulchayanont et al. ${ }^{1,9,16}$ Usually the duration of lesion varies from 1 month to 3 months and in the present case was of two months duration. Patients may present with extraoral or oral lesions, however oral lesions are a rare finding. Most of the oral cases may not be documented in literature due to the least possibility of its occurrence in that area. Diagnosis may be established with FNA, MRI but histopathology still remains gold standard for the diagnosis.

\section{CONCLUSION}

Many a times a benign looking cystic lesion in the oral cavity escapes the diagnosis of parasitic cyst which should be treated with medications along with surgical exploration. Thus, cysticercosis should be included in the differential diagnosis of all oral nodular lesions in all patients, especially in developing and endemic countries and all oral cases should be documented owing to its rare occurrence.

\section{REFERENCES}

1. N. J. Mazhari, Neeta Kumar, Shyama Jain. Cysticercosis of the oral mucosa: aspiration cytologic diagnosis. J Oral Pathol Med 2001,30: 187-9.

2. Wadia, NH, Singh, G. "Taenia Solium: A Historical Note" Taenia Solium Cysticercosis: From Basic to Clinical Science CABI Publishing, 2002. 157-168.

3. Cox, F.E.G. "History of Human Parasitology" Clinical Microbiology Reviews. October 2002. 15(4) 595-612.

4. Ogilvie CM, Kasten P, Rovinsky D, Workman KL, Johnston JO. Cysticercosis of the triceps - an unusual pseudotumor: case report and review. Clin Orthop Relat Res 2001; 382: 217-21.

5. Abdelwahab IF, Klein MJ, Hermann G, Abdul-Quader M. Solitary cysticercosis of the biceps brachii in a vegetarian: a rare and unusual pseudotumor. Skeletal Radiol 2003; 32: 424-8.

6. Jankharia BG, Chavhan GB, Krishnan P, Jankharia B. MRI and ultrasound in solitary muscular and soft tissue cysticercosis. Skeletal Radiol 2005; 34:722-6.

7. Nigam S, Tejinder S, Mishra A, Chaturbedi KU. Oral cysticercosis - report of six cases. Head neck 23: 497-499, 2001. 
8. Fazakerley MW, Woolgar JA. Cysticercosis cellulosae: an unusual cause of a labial swelling. Br Dent J 1991; 170: 105106.

9. Saran RK, Rattan V, Rajwanshi A, Nikjawan R, Gupta SK. Cysticercosis of the oral cavity. Report of five cases and a review of literature. Int J Pediatr Dent 1998;8: 273-278.

10. Lustman J, Coplyn M. Oral cysticercosis: a review of literature and report of two cases. Int J Oral Surg 1981;10: 371375.

11. Ostrofsky MK, Baker MA. Oral cysticercosis: three case reports. J Dent Assoc So Afr 1975;30:535-537.

12. Hansen LS, Allard RHB. Encysted parasitic larvae in the mouth. J Am Dental Assoc1984;108: 632-636.371-375.
13. Amatya, Birendra M. M.B.B.S.; Kimula, Yuji M.D., Ph.D. Cysticercosis in Nepal: A Histopathologic Study of Sixty-two Cases, The American Journal of Surgical Pathology Issue: Volume 23(10), October 1999, p 1276.

14. Kamal MM, Grover SV. Cytomorphology of subcutaneous cysticercosis. A report of 10 cases. Acta Cytol 1995; 39: 809-12. 15. Elias FM, Martins MT, Foronda R, Jorge WA, Araújo NS. Oral cysticercosis: case report and review of the literature. Rev Int Med Trop São Paulo 2005;47:95-8.

16. Vorachai Sirikulchayanonta, Suphaneewan Jaovisidha MD. An Intramuscular Cysticercosis, A Case Report with Correlation of Magnetic Resonance Imaging and Histopathology. J Med Assoc Thai 2007; 90 (6): 1248-52. 